\title{
The interaction of psychogenic polydipsia with wheel running in rats'
}

EVALYN F. SEGAL, University of Illinois at Chicago Circle, Chicago, Ill. 60680 .

Rats on a free-food schedule had access both to a water bottle and to a running wheel. As schedule-induced polydipsia developed, amount of running also increased, but drinking held primacy in three of four rats. When drinking was prevented, running assumed a temporal pattern similar to that characteristic of schedule-induced polydipsia.

If rats are given spaced dry food pellets, either as reinforcers for a selected operant or on a free-food schedule, a typical pattern of behavior develops. Immediately after eating a food pellet the rat goes to the water bottle and drinks for some seconds before shifting to other behavior. The amount of water consumed is much higher than normally seen in either ad lib or food-deprived rats. Rats on ad lib food rations drink about 16-41 ml of water daily (Falk, 1961; Stellar \& Hill, 1953), and much less when they are on restricted food rations (Falk, 1964, 1967; Kutscher, 1967; Schaeffer \& Salzberg, 1967; Verplanck \& Hayes, 1953). In contrast, polydipsic rats drink about 40-100 ml during 3-h sessions of spaced feeding (Falk, 1961, 1964; Schaeffer \& Diehl, 1966; Segal \& Holloway, 1963). Moreover, they drink more, not less, water as the amount of food offered per portion decreases (Falk, 1967). Whereas ad lib rats consume water to food in a weight ratio of about 2:1 (Falk, 1964) and food-deprived rats in a ratio of about 1:1 (Stricker \& Adair, 1966), polydipsic rats drink seven to 11 times as much water (by weight) as food (Falk, 1964; Stricker \& Adair, 1966).

Why rats drink excessively when exposed to a spaced-food schedule is not clear. One possibility is that drinking is simply
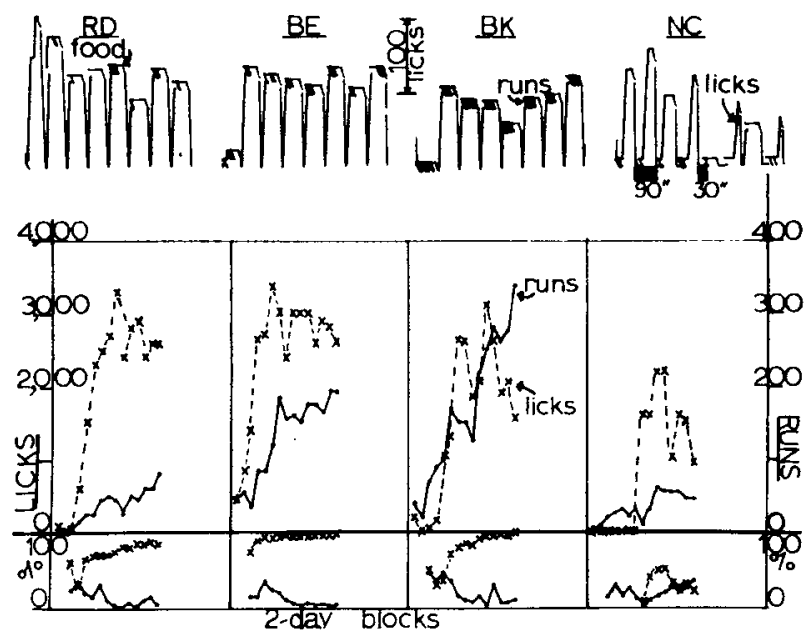

Fig. 1. Results of the first 29 days of the experiment. Top: licks stepped the recording pen upward, and wheel revolutions deflected the pen upward or downward. The pen was deflected downward during the first $30 \mathrm{sec}$ of each 90 -sec interpellet interval. Sample intervals are shown for each rat. Middle: runs (solid line) and licks (dashed line) per session, averaged over two-day blocks (except the last, odd-numbered day). Note that the ordinate scales for running and licking are different. Bottom: percentage of all rums (solid line) and all licks (dashed line) that occurred in the first $30 \mathrm{sec}$ of each $90 \mathrm{sec}$ interval, over the first 29 days, averaged in two-day blocks (except the last, odd-numbered day). something to do to pass the time while waiting for the next food pellet. If that is so, then the opportunity to engage in another behavior with a high base rate ought to compete with, and eliminate or reduce, polydipsia, Levitsky (1967) reported wheel-running behavior in rats that resembled scheduleinduced polydipsia, which may support the something-to-do hypothesis. However, Levitsky's report did not mention whether running supplemented drinking or replaced it, or what interaction existed between the two responses under conditions where both might occur. The present experiment addressed that question.

Four adult, male, albino rats, maintained at $80 \%$ of ad lib weight by food but not water deprivation, were given 25 Noyes 45 -mg rat chow pellets at a spacing of one every $90 \mathrm{sec}$ in an experimental chamber that included a depressible bar, a water bottle, and a door that gave access to a running wheel. Bar presses, water licks, and wheel revolutions were recorded, but the arrival of food was contingent on none of these responses. This phase of the experiment lasted 29 days.

Three of the rats were then run for 83 days in another experiment (Segal \& Oden, in press), in the course of which they were given occasional foot shocks in the same chamber and while the same food schedule continued in effect. However, so that the rats could not escape scheduled shocks, the door to the running wheel was closed, barring access to the wheel.

On the day following completion of the shock experiment, the door to the running wheel was opened, again permitting the rats to run. The next day, access to the running wheel was barred again, but on the succeeding three days running was permitted. On the final day, the water bottle was removed, so that drinking could not occur.

\section{RESULTS}

Figure 1 shows data for the first phase of the experiment, which preceded the shock experiment. The top part of the figure shows sample cumulative records. During the first $30 \mathrm{sec}$ of each interpellet interval the recording pen was offset downward, and wheel revolutions recorded as brief upward deflections of the pen. During the last $60 \mathrm{sec}$ of each interval the pen was in "normal" position, and wheel revolutions recorded as brief downward deflections of the pen. In either case, water licks stepped the pen upward. The pen reset to baseline following each pellet delivery. Bar presses were rare or nonexistent, and so the graphic record of bar presses is not included in the figure.

Note the pattern of drinking and running. Following each pellet delivery, Rats RD, BE, and BK generally took a drink of water lasting about $30 \mathrm{sec}$. Then they entered the wheel and ran for varying durations. This pattern varied only occasionally. Rat NC, on the other hand, rarely ran, and when it did, it ran before, not after, drinking.

The middle part of Fig. 1 shows the development over days of the drinking-running pattern. The solid line shows the total number of wheel revolutions, averaged for 2-day blocks (except the last, odd-numbered day), and the dashed line the total number of water licks. Note that the ordinate scales for running and drinking differ by a factor of 10 . Both licks and runs increased to more or less stable asymptotes over the 29 days of this phase of the experiment.

The bottom part of Fig. 1 shows the percentage of all runs and all licks that occurred in the first $30 \mathrm{sec}$ of the 90 -sec interpellet interval. The solid line represents runs and the dashed line licks. As the experiment progressed, the percentage of all runs that occurred in the first $30 \mathrm{sec}$ of the interval decreased, while the percentage of all licks that occurred in the same period increased, for all rats except Rat NC. Except in Rat NC, licking came more and more to be confined to the 

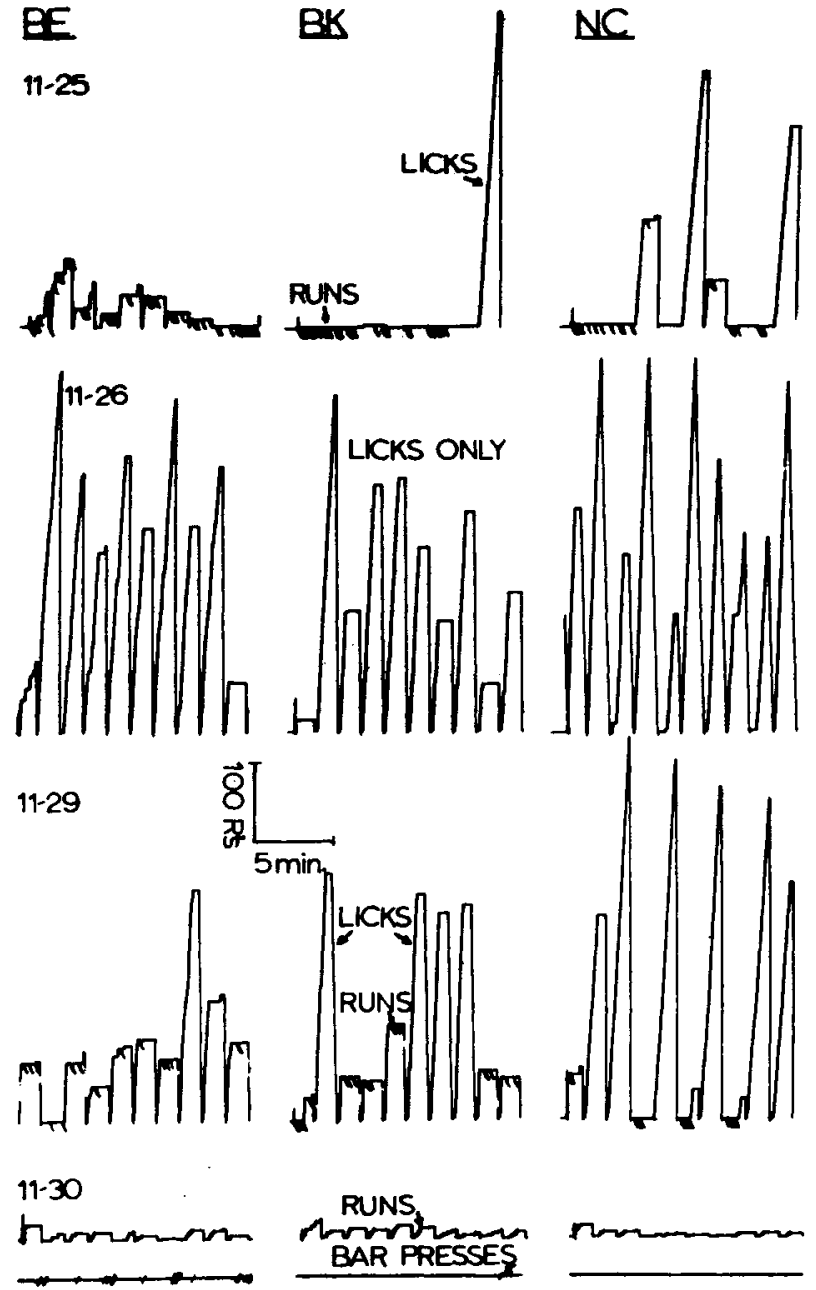

Fig. 2. Results of the second phase of the experiment. Top: runs (downward deflections of the pen) and licks (steps upward of the pen) during the first session in 84 days in which running was permitted. Food deliveries reset the pen to baseline. Second set of records: licks in the next session, in which running was not permitted. Third set of reconds: licks and runs in the third of three consecutive sessions in which both running and licking were permitted. Bottom: runs during one session in which licking was not permitted. Runs now stepped the recording pen upward. The event line below shows bar presses.

30-sec period immediately after food delivery, and running to the final $60 \mathrm{sec}$ of the interpellet interval.

These data tell us that for most rats, drinking holds primacy over running; that is, drinking occurs first. The data do not tell us whether the opportunity to run decreased the amount of drinking, or whether the opportunity to drink decreased the amount of running. That is, they do not indicate whether there was competition between the two responses. Data relevant to this point appear in Fig. 2. These data were recorded after the intervening 83-day shock experiment was completed. Note that in Fig. 2 the recording pen no longer offset downward during the first $30 \mathrm{sec}$ of each $90-\mathrm{sec}$ interpellet interval.

The top set of records was obtained on the first day of this new phase of the experiment. It shows performance during the first session in 84 days in which running was again permitted. Note that the three rats who continued into this phase of the experiment spent most of the session running, not drinking. Far less drinking occurred than during any previous session of the experiment, including the interpolated shock sessions.
During subsequent sessions in which running and drinking were both permitted, drinking recovered to higher levels (see the third set of records in Fig. 2). It seems likely, therefore, that the prepotency of running over drinking evident in the top set of records was the result of the relative novelty of running (the rats had not been permitted to run for the preceding 83 days).

The second set of records in Fig. 2 shows performance on the next day, when (by experimental error, not design) the door to the running wheel was again closed. All three rats spent a large proportion of their session time drinking. This performance was typical of that seen during the intervening shock experiment.

The third set of records shows performance during the third of three consecutive sessions in which both running and drinking were permitted. The pattern is much like that seen in Fig. 1; drinking occurred following every food delivery, giving way after some seconds to running, in Rats BE and BK. Running preceded drinking, on those occasions when running occurred at all, in Rat NC.

The number of licks in the "licks only" session (11/26) was 6301 by Rat BE, 3495 by Rat BK, and 6812 by Rat NC. The number of licks in the succeeding three sessions of running and licking was: $2738,2788,2913$ in Rat BE; 3650, 3552, 2659 in Rat BK; and 4614, 5055, 5140 in Rat NC. These data suggest that running competed with licking, at least in rats $\mathrm{BE}$ and $\mathrm{NC}$; there was more licking by rats $\mathrm{BE}$ and $\mathrm{NC}$ when running was not available.

The bottom set of 'records shows running and bar pressing on the final day of the experiment, when the water bottle was removed so that drinking could not occur. Note that the manner of recording running was different here than earlier. Running now stepped the recording pen upward, as licking had done previously. This change was made to permit an easy comparison of the temporal pattern of running, when running did not compete with licking, and the temporal pattern of licking seen earlier.

The noteworthy finding is that when running was not in competition with licking it assumed a temporal pattern comparable to the pattern of polydipsic licking. That is, running began soon after the arrival of the food pellet, and continued for some seconds, generally ceasing some seconds before the next pellet arrived. Far fewer wheel revolutions occurred in this session than water licks in earlier sessions, but this difference in scale does not detract from the similarity of the temporal patterns.

The number of wheel revolutions in the final session, when licking could not occur, was 219 for Rat BE, 186 for Rat BK, and 283 for Rat NC. The number of revolutions for each of the preceding three sessions, in which both licking and running could occur, was: $192,205,124$ in Rat BE; 205, 148, 195 in Rat BK; and 101, 103, 138 in Rat NC. Only for Rat NC was there clear evidence that licking competed with running; there was more running by Rat NC when licking was not available.

The last session of the experiment was the only session in which appreciable amounts of bar pressing occurred, and so is the only session for which the graphic records of bar pressing are shown.

It appears, then, that when rats are exposed to a spaced food schedule in a chamber that permits drinking, running, and bar pressing, licking is the predominant response, running next, and bar pressing last. In one respect, running may be substituted for licking; if licking cannot occur, running occurs earlier. In another respect, the two responses do not appear to be strictly interchangeable; preventing licking does not necessarily increase the amount of running, although preventing running does generally increase the amount of licking. These data do not appear to fit a simple "something to do" account of psychogenic polydipsia. Neither do they fit a 


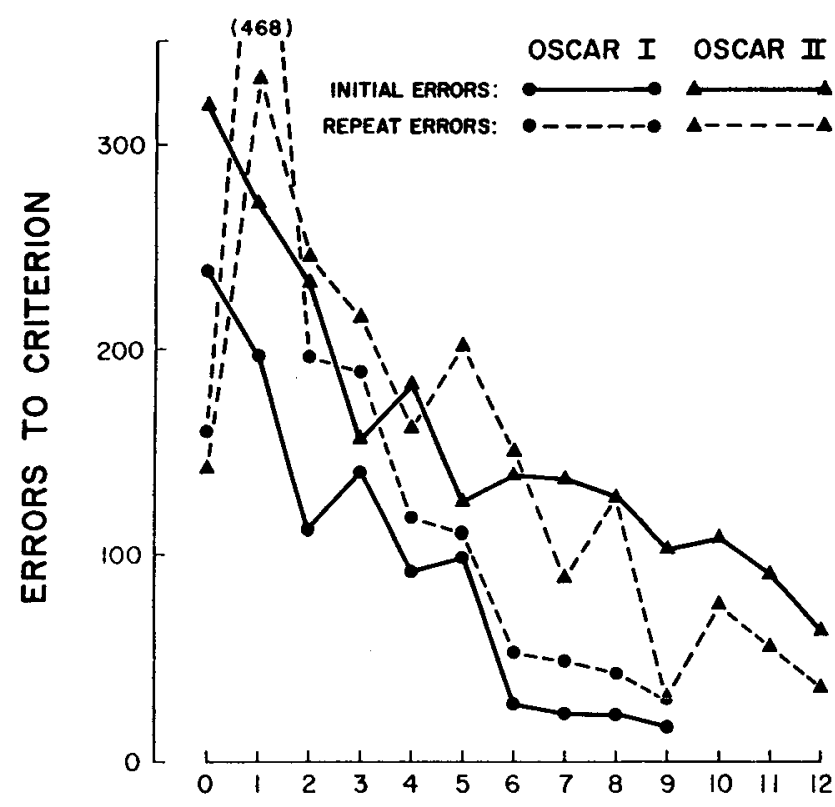

REVERSAL

Fig. 2. Number of sessions required to reach criterion of $96 \%$ for each S. Each reversal session consisted of $\mathbf{5 0}$ correct discriminations, plus errors.

vibration from the feeder, but there was no evidence that it was aversive after the first day. Regular target responding was established in $3 \mathrm{~h}$ without deprivation or shaping. Thereafter, all food was provided in experimental sessions, save for an occasional feeding of angleworms on a nonexperimental day.

A criterion of 96 per cent correct, not more than two errors in $\mathbf{5 0}$ presentations, indicated that the problem was mastered thoroughly before reversal. Both Oscars completed some sessions without error. S1 jumped from its tank despite a glass cover after Reversal 9 and S2 died from an undiagnosed ailment in the midst of Reversal 13.

\section{RESULTS}

Figure 1 shows that both $\mathrm{S} 1$ and S2 steadily improved, making both fewer initial and fewer repetitive errors on later reversals. Fig. 2 shows the number of sessions required to reach criterion by each $S$. Both Ss continued to improve, although neither acquired a correct performance within a few trials, despite the length of the study.

The Oscar may be peculiarly suited to a visual reversal task, producing results superior to other fish species, or, as Setterington \& Bishop (1967) suggest, the Ss may not be representative for their species. Since so few fish species have been used in studies of reversal learning, it is premature to conclude that the Oscar, mouthbreeder, or goldfish is representative of its class (Bitterman, 1965). Mackintosh \& Mackintosh (1964) have shown that cephalopods, evolved in aquatic environments, do improve in reversal learning, so many fish species may have evolved with such potential.

Methodological differences which may account for the differences in results between this and other studies include the use of unscreened tanks with visual access to other fish, constant background of illumination, and large home tanks with no moving of the tank or fish. The use of dry pellets instead of worms as reinforcers is an unlikely cause of the demonstrated improvement, since Oscars do prefer live food.

Clearly, fine-grain analysis of fish behavior in the complex reversal situation is necessary to identify the controlling variables. The improved Ames feeder (1967) and target system (unpublished) will simplify this task, both because of their high reliability and because they permit much more data to be collected per $S$ daily. We are now using this refined equipment.

\section{REFERENCES}

AMES, L. L. A liquid-food, limited-access feeder for fish. Journal of the Experimental Analysis of Behavior, 1967, 10, 435-437.

BEHREND, E. R., \& BITTERMAN, M. E. Further experiments on habit reversal in the fish. Psychonomic Science, 1967, 8, 363-364.

BITTERMAN, M. E. Phyletic differences in learning. American Psychologist, 1965, 20, 396410.

HEMMINGS, G., \& MATTHEWS, W. A. Shape discrimination in tropical fish. Quarterly Journal of Experimental Psychology, 1963, 15, 273-278.

LISSMANN, H. W. Die Umwelt des Kampffishes. Zeitschrift für vergleichende Physiologie, 1932, 18, 62-111.

MACKINTOSH, N. J., \& MACKINTOSH, J. Performance of Octopus over a series of reversals of a simultaneous discrimination. Animal Behavior, 1964, 12, 321-324.

MARK, R. F., \& DAVIDSON, T. M. Unit responses from commissural fibers of optic lobes of fish. Science, 1966, 152, 797-799.

MARRONE, R., \& EVANS, S. Two-choice and three-choice probability learning in fish. Psychonomic Science, 1966, 5, 327-328.

SETTERINGTON, R. G., \& BISHOP, H. E. Habit reversal improvement in the fish. Psychonomic Science, 1967, 7, 41-42.

(Continued from page 142)

simple "thirst" account. Possibly both responses may represent emotional arousal generated by the "frustration" of waiting for small food portions to be doled out at spaced intervals. The primacy of licking may represent the interaction of "thirst" with emotional arousal.

\section{REFERENCES}

FALK J. L. Production of polydipsia in normal rats by an intermittent food schedule. Science, 1961, 133, 195-196.

FALK, J. L. Studies on schedule-induced polydipsia. In M. J. Wayner (Ed.), Thirst. New York: Pergamon Press, 1964. Pp. 95-116.

FALK, J. L. Control of schedule-induced polydipsia: Type, size, and spacing of meals. Journal of the Experimental Analysis of Behavior, 1967, 10, 199-206.

KUTSCHER, C. L. Species differences in the interaction of feeding and drinking. Paper presented at the Conference on Feeding and Drinking, New York Academy of Sciences, February, 1967.

LEVITSKY, D. A. Psychogenic wheel running. Psychonomic Bulletin, 1967, 1, 35. (Abstract)

SCHAEFFER, R. W., \& DIEHL, J. C. Collateral water drinking in rats page 142) on FR food reinforcement schedules. Psychonomic Science, 1966, 4, 257-258.

SCHAEFFER, R. W., \& SALZBERG, C. L. Schedule-induced polydipsia: An aty pical case. Psychological Reports, 1967, 20, 1071-1076.

SEGAL, E. F., \& HOLLOWAY, S. M. Timing behavior in rats with water drinking as a mediator. Science, 1963, 140, 888-889.

SEGAL, E. F., \& ODEN, D. L. Effects of drinkometer current and of foot shock on psychogenic polydipsia. Psychonomic Science, in press.

STELLAR, E., \& HILL, J. H. The rat's rate of drinking as a function of water deprivation. Journal of Comparative \& Physiological Psychology, 1952, 45, 96-102.

STRICKER, E. M., \& ADAIR, E. R. Body fluid balance, taste, and postprandial factors in schedule-induced polydipsia. Journal of Comparative \& Physiological Psychology, 1966, 62, 449-454.

VERPLANCK, W. S., \& HAYES, J. R. Eating and drinking as a function of maintenance schedule. Journal of Comparative \& Physiological Psy chology, 1953, 46, 327-333.

\section{NOTE}

1. This experiment was performed at San Diego State College, and supported by National Science Foundation Grants NSF GB 1605, NSF GB 5777, and NSF GB 7292. I thank William Martin, David Brookshire, and Michael Turner for their assistance in running the experiment. 